\title{
EPIs e segurança dos trabalhadores de saúde durante a pandemia de COVID-19:
}

\section{Uma breve reflexão}

\author{
PPE and healthcare workers safety during the COVID-19 pandemic: A brief reflection \\ EI EPP y la seguridad de los trabajadores de la salud durante la pandemia del COVID-19: Una \\ breve reflexión
}

Recebido: 04/06/2021 | Revisado: 14/06/2021 | Aceito: 18/06/2021 | Publicado: 02/07/2021

Fábio José Soares Cavalcante

ORCID: https://orcid.org/0000-0001-5975-8244 Centro Universitário UNINOVAFAPI, Brasil E-mail: fcavalcantemeu@gmail.com

Ianny Raquel Dantas Nascimento ORCID: https://orcid.org/0000-0002-3904-5252 Centro Universitário UNINOVAFAPI, Brasil E-mail: iannycel123@gmail.com

Regina Márcia Soares Cavalcante ORCID: https://orcid.org/0000-0002-0500-9990 Universidade Federal do Piaú, Brasil E-mail: reginalunna@hotmail.com

\begin{abstract}
Resumo
A atual pandemia causada pelo novo coronavírus, tem representado um dos mais graves problemas de saúde pública mundiais de todos os tempos. Para o enfrentamento da COVID-19 foram necessárias várias reformulações e adaptações no setor saúde para enfrentamento desta doença de elevado poder de contágio e com números tão elevados de letalidade. $\mathrm{O}$ objetivo deste trabalho foi realizar um levantamento bibliográfico para estudar a utilização de EPIs para segurança dos profissionais e trabalhadores de saúde no período de pandemia de COVID-19. Uma das principais imposições desta nova realidade foi a demanda aumentada de atendimento nos estabelecimentos de saúde, aumentando dessa forma o risco para os profissionais e trabalhadores de saúde em desenvolver as doenças infecciosas como a COVID-19, quando comparado a população em geral, devido a maior chance de exposição a carga viral durante o período de trabalho. Em doenças como a COVID-19, além da transmissão por gotículas e contato, há exposição a procedimentos geradores de aerossóis durante a assistência aos pacientes, e os profissionais da saúde podem agir como potenciais transmissores assintomáticos, infectando outros pacientes, profissionais e familiares. Nesse contexto os equipamentos de proteção individual constituem-se em ferramenta eficaz para a prevenção de infeção e redução do contágio pela COVID-19.
\end{abstract}

Palavras-chave: EPIs; Trabalhadores de saúde; Segurança no trabalho; COVID-19.

\begin{abstract}
The current pandemic caused by the new coronavirus, has represented one of the most serious public health problems in the world of all time. To cope with COVID-19, several reforms and adaptations in the health sector were necessary to face this disease with high contagion power and with such high numbers of lethalities. The objective of this work was to carry out a bibliographic survey to study how the use of PPE for the safety of health professionals and workers in the pandemic period of COVID-19. One of the main impositions of this new reality was the increased demand for care in health establishments, thus increasing the risk for health professionals and workers to develop infectious diseases such as COVID-19, when compared to the general population, due to greater chance of exposure to viral load during the work period. In diseases such as COVID-19, in addition to droplet transmission and contact, there is exposure to aerosol-generating procedures during care for these patients, and health professionals can act as potential asymptomatic transmitters, infecting other patients, professionals and family members. In this context, personal protective equipment is an effective tool for preventing infection and reducing contagion by COVID-19.
\end{abstract}

Keywords: PPE; Healthcare workers; Safety at work; COVID-19.

\section{Resumen}

La pandemia actual provocada por el nuevo coronavirus, ha representado uno de los problemas de salud pública más graves del mundo de todos los tiempos. Para hacer frente al COVID-19 fueron necesarias varias reformas y adaptaciones en el sector salud para enfrentar esta enfermedad con alto poder de contagio y con tan alto número de letalidades. El objetivo de este trabajo fue realizar un relevamiento bibliográfico para estudiar cómo se está produciendo 
el uso de EPP para la seguridad de los profesionales y trabajadores de la salud en el período pandémico de COVID-19. Una de las principales imposiciones de esta nueva realidad fue la mayor demanda de atención en los establecimientos de salud, aumentando así el riesgo de que los profesionales y trabajadores de la salud desarrollen enfermedades infecciosas como COVID-19, en comparación con la población general, debido a una mayor probabilidad de aparición de enfermedades infecciosas. exposición a la carga viral durante el período de trabajo. En enfermedades como el COVID-19, además de la transmisión por gotitas y contacto, existe exposición a procedimientos generadores de aerosoles durante el cuidado de estos pacientes, y los profesionales de la salud pueden actuar como potenciales transmisores asintomáticos, infectando a otros pacientes, profesionales y familiares. En este contexto, el equipo de protección personal es una herramienta eficaz para prevenir la infección y reducir el contagio por COVID-19.

Palabras clave: EPP; Trabajadores de la salud; Seguridad en el trabajo; COVID-19.

\section{Introdução}

A doença coronavírus em 2019 - COVID-19, causada pelo novo coronavírus, originou-se na China, na cidade de Wuhan em dezembro de 2019 e, em decorrência do elevado poder de disseminação da doença, tornou-se, em apenas três meses, uma pandemia muito impactante de forma generalizada por todo o globo e, tem se mostrado como o maior problema de saúde pública em todo o mundo, marcando definitivamente a história da humanidade (Lipsitch, Swerdlow \& Finelli, 2020).

Conforme as informações atualmente disponíveis, a via de transmissão pessoa a pessoa do SARS-CoV-2 ocorre por meio de gotículas respiratórias (expelidas ao falar, tosse ou espirro) e também pelo contato direto com pessoas infectadas ou indireto por meio das mãos, objetos ou superfícies contaminadas, de forma semelhantes com que outros patógenos respiratórios se disseminam (Anvisa, 2020).

Diante da emergência na saúde pública mundial surgiu, de forma crescente, a demanda de trabalhadores da área de saúde, como como médicos, enfermeiros, fisioterapeutas, técnicos de enfermagem, agentes comunitários de saúde, pessoal de limpeza, porteiros e atendentes de serviços de saúde, para compor a linha de frente no enfrentamento da COVID-19. Estes trabalhadores da saúde, estão mais expostos a contrair a doença, por estarem em contato direto com pessoas contaminadas. No caso específico de trabalhadores /profissionais de saúde, segundo dados disponíveis, foi estimado que na China, mais de três mil profissionais tenham se infectado com o coronavírus, dos quais 23 morreram (Xiang et al., 2020). Na Itália, 4.884 casos de COVID-19 ocorreram entre profissionais de saúde, com 24 óbitos dentre os médicos (Anelli et al., 2020).

Como a COVID-19 é uma doença ainda nova e por não haver tratamento de eficácia comprovada para a mesma, as estratégias de distanciamento social têm sido apontadas como as mais importantes intervenções na prevenção e controle da doença. Entretanto, para as equipes de assistência à saúde, especialmente aqueles profissionais atuantes no combate primário à pandemia, que estão envolvidos no cuidado direto de casos suspeitos e diagnosticados de COVID-19 nos serviços de atenção primária, nas unidades de pronto-atendimento e nos hospitais, é inviável a prática desta recomendação de permanecer em casa, o que torna este grupo de profissionais e trabalhadores mais vulneráveis para contaminação com o novo coronavírus (Teixeira et al., 2020) .

É importante enfatizar que as infecções por COVID-19 estão associadas à inadequação ou a falhas nas medidas de precaução e de proteção contra a doença, falta de equipamentos de proteção individual - EPIs (máscaras cirúrgicas e do tipo PFF2 e vestuário), presença de aglomerações, indivíduos infectados e assintomáticos que mantiveram contato com profissionais e demais trabalhadores de saúde, dentre outros fatores (Xiang et al., 2020).

Nessa perspectiva, o objetivo deste trabalho foi estudar a utilização de EPIs para segurança dos profissionais e trabalhadores de saúde no período de pandemia de COVID-19.

\section{Metodologia}

Estudo de natureza qualitativa e exploratória, desenvolvido por meio de uma revisão de literatura narrativa (Estrela, 2018), elaborada por meio da busca de artigos científicos nas bases de dados Pub Med, Scielo e Scopus utilizando como 
descritores: COVID-19; PPE; healthcare workers e safety at work e, correspondentes em português COVID-19; EPIs; trabalhadores de saúde e segurança no trabalho. Foram incluídos no estudo artigos em inglês e português disponíveis na íntegra e que apresentaram abordagens relevantes para o tema proposto. Foram excluídos aqueles artigos que se repetiam nas bases de dados analisadas, bem como, mediante leitura do resumo ou artigo completo, os que não estavam condizentes com o tema abordado. Quanto a interpretação de dados, esta foi realizada a luz da literatura cientifica disponível, reafirmando que os resultados encontrados atendiam aos objetivos propostos nesse estudo.

\section{Resultados e Discussão}

Um novo tipo de coronavírus, denominado SARS-CoV-2, foi identificado em Wuhan, China, em dezembro de 2019, e levou a um surto de rápida disseminação da doença coronavírus em 2019 (COVID-19). Logo em seguida, no dia 30 de janeiro de 2020, o COVID-19 foi declarada uma emergência de saúde pública de interesse internacional (Chan et al., 2020). No dia 11 de março a Organização Mundial da Saúde (OMS) declarou a doença como uma pandemia (WHO, 2020; OPAS, 2020). No Brasil, o primeiro caso da doença foi confirmado em 26 de fevereiro de 2020 e, em 22 de maio tornou-se o país com o segundo maior número de casos da doença no mundo (Word O’ Meters, 2020).

São considerados importantes sinais e sintomas da COVID-19: febre, tosse seca, dispneia, mialgia ou fadiga e linfopenia. A transmissão da doença é realizada por meio de gotículas - contato interpessoal íntimo e, dessa forma, as secreções respiratórias foram consideradas o principal meio de propagação do vírus. A análise de cultura de células epiteliais das vias aéreas humanas, a microscopia eletrônica e o sequenciamento completo do genoma da cultura sobrenadante foram compartilhados com a OMS, destinando-se à vigilância e deteç̧ão da infecção por 2019-nCoV, na China e globalmente (Huang, Wang, Li, Ren, Zhao \& Hu, 2020). Além disso, tem-se analisado a possibilidade de transmissão do vírus por aerossóis (partículas menores e mais leves que as gotículas), gerados durante alguns procedimentos específicos (Avisa, 2020). O SARSnCoV-2 pode ser transmitido por meio de menores aerossóis com núcleos de gotículas $\leq 5 \mu \mathrm{m}$, que podem viajar longas distâncias e permanecer no ar por 2 a 4 horas, dependendo das condições ambientais (Van Doremalen, 2020).

Considerando a transmissibilidade, o principal problema de saúde que atinge os profissionais envolvidos na linha de frente do cuidado aos pacientes sintomáticos ou diagnosticados com COVID-19 é o risco de contaminação pela doença. (Holland \& Friderich, 2020). Nesse contexto é importante enfatizar que considerando o ritmo lento da vacinação no Brasil, os profissionais de saúde não podem, pela natureza do seu trabalho, dispor dos meios mais eficazes na prevenção de contaminação pela COVID-19, que são o confinamento e o isolamento social (Brito et al., 2021). Há muitas evidências que indicam o alto grau de exposição e contaminação dos profissionais de saúde pelo novo coronavírus (Ran et al.,2020), que por ser um vírus de transmissão respiratória, encontra na utilização de EPIs, ação indispensável como medida de precaução padrão, de contato e por gotículas recomendadas no enfrentamento desta pandemia (Holland \& Friderich, 2020)

Dentro desse contexto, os profissionais e trabalhadores de saúde constituem grupo de risco para a COVID-19 e, a segurança no ambiente de trabalho e consequente proteção dos mesmos tem papel de extrema relevância para evitar a transmissão e minimizar os risco de infecção pela doença, tanto nos estabelecimentos de saúde quanto em suas residências, tornando-se essencial a adoção de protocolos de controle de infecções (padrão, contato, via aérea) como também a disponibilização de EPIs, como máscaras N95, aventais, óculos, protetores faciais e luvas (Teixeira et al., 2020).

De acordo com a Agência de Vigilância Sanitária-ANVISA (2020) é fundamental no período pandêmico de COVID19, que os trabalhadores de saúde, que estão trabalhando diretamente no enfrentamento da doença, tenham acesso, bem como conhecimento sobre a importância do uso de EPIs para a prevenção de contaminação e redução na transmissão de microorganismos durante a assistência aos pacientes. 
Durante o surto inicial de COVID-19, em Wuhan, 13 profissionais de saúde foram infectados (Gawande, 2020). Eles se tornaram os vetores de transmissão para seus colegas e familiares, e 42.000 profissionais de saúde tiveram que ser trazidos para tratar os pacientes, pois os profissionais de saúde sucumbiram ao COVID-19 (Zang et al., 2020). Nesse contexto, as medidas de prevenção e controle devem ser implementadas em todas as etapas do atendimento do paciente no serviço de saúde, desde sua chegada, triagem, espera, durante toda a assistência prestada, até sua a sua alta/transferência ou óbito (Anvisa, 2020). Em setembro de 2020, o Escritório Regional Pan-Americano da OMS em Washington, DC, informou que 570.000 profissionais de saúde estavam infectados e 2.500 morreram devido ao COVID-19 nas américas (OPAS /WHO, 2020; WHO, 2020a) e, milhares de profissionais de saúde infectados com a doença, perderam suas vidas em todo o mundo (WHO, 2020b)

De acordo com a norma regulamentadora (NR) 6 do Ministério do Trabalho, entende-se por equipamento de proteção individual (EPI) "todo dispositivo ou produto, de uso individual, utilizado pelo trabalhador, destinado a proteção de riscos, à segurança e saúde no trabalho". No que se refere à especificação do uso de EPIs em ambiente hospitalar, a ANVISA, por meio das Notas Técnicas 04/2020 e 07/2020, definiu as recomendações, considerando o risco de exposição do profissional de saúde ao material biológico, estendendo-se da precaução padrão para situações específicas, como a definida para o atendimento aos pacientes com COVID-19 (Anvisa, 2020a; Anvisa, 2020b; Ministério do Trabalho, 2020). É importante enfatizar que a utilização de EPIs, é considerada uma das estratégias mais importantes para proteger pacientes e profissionais de saúde de patógenos transmissíveis (Bettini, Ramos \& Almeida, 2020; Honda \& Kentaro, 2016), a exemplo do novo coronavírus.

Estudo realizado em 205 pacientes com COVID-19, analisou 1070 amostras coletadas de locais variados identificou a presença do vírus em: lavado broncoalveolar (93\% das amostras), escarro (72\%), swab nasal (63\%), biopsia por fibrobroncoscopia (46\%), swab faríngeo (32\%), fezes (29\%), sangue (1\%) e zero em amostras de urina (Van Doremalen et al., 2020). Considerando estes achados é evidenciado que os EPIs devem ser utilizados por todos os profissionais de saúde, em todos os momentos da assistência aos pacientes com suspeita ou diagnosticados com COVID-19, quando exista possibilidade de contato com esses materiais (WHO, 2020).

Trabalhadores dos serviços de saúde são todos aqueles que atuam em espaços e estabelecimentos de assistência e vigilância à saúde, sejam eles hospitais, clínicas, ambulatórios e outros locais (Anvisa, 2020). Desta maneira, compreende tanto os profissionais da saúde - como médicos, enfermeiros, técnicos de enfermagem, nutricionistas, fisioterapeutas, etc. quanto os trabalhadores de apoio, como recepcionistas, seguranças, pessoal da limpeza, cozinheiros, entre outros, ou seja, aqueles que trabalham nos serviços de saúde, mas que não estão prestando serviços direto de assistência à saúde das pessoas (Ministério da Saúde, 2020).

Para garantia dos direitos dos trabalhadores a Constituição Federal Brasileira de 1988 (Brasil,1988) e a Convenção no 155 (OIT, 1981), da Organização Internacional do Trabalho (OIT) incorporadas pela legislação do Brasil, definem que toda empresa ou organização tem responsabilidade referente à saúde e segurança do trabalhador e de outros que possam ser afetados por suas atividades. Aliado a estes dois amparos legais, a Lei Orgânica do Sistema Único de Saúde - SUS, nº 8.080, de 19 de setembro de 1990 (Brasil, 1990), garante a promoção e proteção da saúde dos trabalhadores submetidos aos riscos e agravos advindos das condições de trabalho, bem como a recuperação, reabilitação e assistência às vítimas de acidentes doenças e agravos relacionados ao trabalho.

Neste sentido, todos os serviços de saúde devem garantir a adoção de medidas e mecanismos de proteção e promoção à saúde para todos os trabalhadores que atuam nos serviços, sejam ele empregados, terceirizados ou pertencentes a outras modalidades de vínculos. De forma geral, a Norma Regulamentadora de Segurança e Saúde no Trabalho em Serviços de Saúde 32 (NR 32), tem como finalidade estabelecer as diretrizes para implementação de medidas básicas de proteção à segurança e à saúde dos trabalhadores dos serviços de saúde e, estabelece que o empregador deve disponibilizar ao trabalhador EPIs em 
quantidade suficiente, descartáveis ou não, sendo essencial para a execução segura das tarefas rotineiras no ambiente de trabalho (Brasil, 2005)

Segundo o Ministério da Saúde (2020), de maneira geral, os EPIs que devem estar disponíveis pelos serviços e utilizados pelos profissionais de saúde que atendem os casos suspeitos ou confirmados de COVID-19 são: 1) gorro; 2) óculos de proteção ou protetor facial; 3) máscara); 4) avental impermeável de mangas compridas; 5) luvas de procedimento. No que se refere ao tipo de máscara, para procedimentos que geram gotículas, deve-se utilizar a máscara cirúrgica e utilizar as de

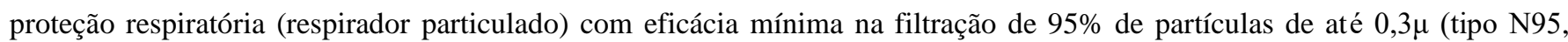
N99, N100, PFF2 ou PFF3), sempre que realizar procedimentos geradores de aerossóis como por exemplo, intubação ou aspiração traqueal, ventilação não invasiva, ressuscitação cardiopulmonar, ventilação manual antes da intubação, indução de escarro, coletas de amostras nasotraqueais e broncoscopias.

Para os profissionais de limpeza, são obrigatórios os seguintes EPIs: luvas de borracha de material resistente, cano longo ou curto para proteção das mãos e proteção parcial de antebraços e as mãos; máscara cirúrgica (exceto em ambientes onde estejam desempenhando atividades com possibilidade de geração de aerossóis). Neste caso, utilizar máscara N95, N99, N100, PFF2 ou PFF3; óculos de proteção; botas de material impermeável, com cano alto e de solado antiderrapante; avental impermeável e gorro.

Em nível mundial, embora não haja uniformidade nas recomendações de EPIs para os profissionais de saúde que atuam na linha de frente de combate à COVID-19, alguns itens são utilizados, de forma homogênea, para o atendimento de casos suspeitos e confirmados da doença, em alguns países como Brasil, Espanha, Itália, China, Estados Unidos, e na Europa de forma geral: luvas descartáveis, roupas de proteção (aventais descartáveis e impermeáveis), máscara cirúrgica, máscara N95, máscara autofiltrante FFP2, máscara autofiltrante FFP3, óculos e viseira (Anvisa , 2020b; CCDC, 2020; CCAES, 2020; CDC, 2020; ECDC, 2020; INAIL, 2020). É importante evidenciar que, de maneira geral, por todos os países, em virtude da demanda crescente de equipamentos de proteção, ocorrido de forma simultânea, nem sempre houve disponibilidade dos EPIs nos estabelecimentos de saúde.

Além disso, é oportuno enfatizar os efeitos indesejáveis relacionados ao uso de EPIs necessários para se evitar ou diminuir os riscos de infecção pelo COVID-19. Estudo desenvolvido por Koh (2020) mostrou elevada incidência de complicações cutâneas associadas com medidas preventivas entre profissionais de saúde que trataram de pacientes com COVID-19, o que pode ocasionar o não uso do EPI em decorrência da ulceração cutânea. Esta pesquisa também revelou que a prevalência de lesões cutâneas relacionadas aos EPIs foi de 97,0\% entre profissionais de saúde da linha de frente e incluíram lesões cutâneas que afetavam a ponte nasal, as mãos, a bochecha e a testa. Além disso, a higienização constante das mãos foi associada a uma maior incidência de dermatite nessa região.

Pesquisa realizada na China por Yan et al. (2020) evidenciou a propensão de lesões na pele e mucosa, decorrentes da inadequação no uso dos EPIs na prevenção e no controle do COVID- 19, sinalizando para a possibilidade dos profissionais de saúde desenvolverem dermatite aguda ou crônica, infecções secundárias e outras doenças de pele. Dentro desse contexto, os especialistas chineses recomendaram aos profissionais de saúde o seguimento rigoroso dos padrões de uso do EPIs, como também a observância de especificações de esterilização e limpeza, impedindo assim a ocorrência de efeitos adversos.

Além desses efeitos, a resistência dos profissionais e trabalhadores de saúde em fazer a utilização dos EPIs, ou usálos de forma inadequada constitui uma grande barreira para prevenir a exposição ao material biológico contaminado com os microrganismos. 


\section{Considerações Finais}

O atual cenário de crise sanitária mundial decorrente da pandemia de COVID-19, representa um dos mais graves problemas de saúde nos últimos tempos. E para o enfrentamento da doença, foi imposta aos profissionais e trabalhadores de saúde uma jornada de trabalho extenuante e perigosa, pelo alto risco de contaminação pela doença que os mesmos estão submetidos diariamente. Para minimizar os riscos de contaminação, o uso de EPIs representa a forma mais eficaz para a segurança no ambiente de trabalho e garantia da continuidade da assistência. Embora não haja uniformidade mundial na recomendação no uso destes equipamentos, os mais frequentemente utilizados são: luvas descartáveis, roupas de proteção (aventais descartáveis e impermeáveis), máscara cirúrgica, máscara N95, máscara autofiltrante FFP2, máscara autofiltrante FFP3, óculos e viseira.

Mesmo sendo garantida por lei, a segurança no ambiente de trabalho em saúde, nem sempre há a disponibilidade de EPIs nos estabelecimentos de saúde, tanto pela demanda aumentada mundialmente por estes equipamentos e consequente escassez em sua oferta, como também pela inabilidade das gestões em saúde no planejamento estratégico para enfrentamento da pandemia de COVID-19 e garantia mínima dos insumos essenciais para o atendimento das pessoas infectadas, nos vários níveis de atenção à saúde. A segurança dos profissionais e trabalhadores de saúde também é comprometida pela falta de adesão ao uso dos EPIs, pelo desconforto provocado pelo uso contínuo ou falta de informação sobre sua importância, bem como pela falta de capacitação destes trabalhadores para o uso adequado dos referidos equipamentos.

Diante da importância dos EPIs para a segurança dos trabalhadores e profissionais da saúde e consequente redução da contaminação pelo novo coronavírus, é de fundamental importância que sejam realizados estudos frequentes e abrangentes, sobre: a oferta destes equipamentos, em qualidade e quantidade adequadas, no ambiente de trabalho; a adesão dos profissionais à utilização destes EPIs, como também sobre a eficiência, eficácia e efetividade da cada item e, assim, fornecer o devido embasamento cientifico para a recomendação mais acertada e padronizada relacionada ao uso destes elementos de segurança.

\section{Referências}

Anvisa - Agência Nacional de Vigilância Sanitária. Serviços de Saúde. http://www.anvisa.gov.br/servicosaude/ organiza/index.htm.

Anvisa a .Agência Nacional de Vigilância Sanitária. Nota Técnica GVIMS/GGTES/ANVISA nº 04/2020. (2020). Orientações para serviços de saúde: medidas de prevenção e controle que devem ser adotadas durante a assistência aos casos suspeitos ou confirmados de infecção pelo novo coronavírus (SARS-CoV-2). <http://portal.anvisa.gov.br/documen ts/33852/271858/Nota+T\%C3\%A9cnica+n+04-2020+G VIMS-GGTES-ANVISA-ATUALIZADA/ab598660-3de4-4f 14-8e6f-b9341c196b28>.

Anvisa b. Agência Nacional de Vigilância Sanitária. Nota Técnica GVIMS/GGTES/ANVISA Nº 07/2020. (2020). Orientações para a Prevenção da Transmissão de Covid-19 dentro dos Serviços de Saúde. 08.05.2020 (Complementar À Nota Técnica Gvims/Ggtes/Anvisa N ${ }^{\circ}$ 04/2020). <http://portal.anvisa.gov. br/documents/33852/271858/NOTA+T\%C3\%89CNICA+-GIMS-GGTES-ANVISA+N\%C2\%BA+07-2020/f487f506 -1eba-451fbccd-06b8f1b0fed6>.

Bettini, N. M. M., Ramos, F..T \& Almeida, P. M. V. (2020). Recomendações de equipamentos de proteção individual no combate ao COVID -19. São Paulo: Rev Recien. 10(31):87-95.

Brasil. Constituição (1988). Constituição da República Federativa do Brasil. Senado Federal: Centro Gráfico, 1988.

Brasil. Lei nº 8.080, de 19 de setembro de 1990. (1990). Lei Orgânica da Saúde.

Brasil. Ministério do Trabalho e Emprego-MTE. Norma Regulamentadora 32 (NR 32) (2005). Diário Oficial da República Federativa do Brasil, <https://www.gov.br/trabalho/pt-br/inspecao/seguranca-e-saude-no-trabalho/normas-regulamentadoras/nr-32.pdf/view>

Brito, B. M. F.; Sousa, A. M.; Alencar, K. C.; Martins, R. M.; Azevedo, R. L. F. \& Araújo, J. M. N. (2021). A pandemia do COVID-19 e um novo problema de saúde pública: Os impactos emocionais em profissionais de saúde. Research, Society and Development, 10(6), E11610615516.

CCAES. Centro de Coordinación de Alertas y Emergencias Sanitarias Ministerio de Sanidad. Gobierno de España. Documento Tecnico. Medidas higiénicas para la prevención de contágios de COVID-19. 2020. <https://www.mscbs.gob. es/profesionales/saludPublica/ccayes/alertasActual/nCovChina/documentos.htm>.

CCDC. Chinese Center for Disease Control and Prevention. (2020) China CDC Weekly. Guidelines for Personal Protection of Specific Groups from COVID19. 2(19):341-343. <http://weekly.chinacd c.cn/en/article/doi/10.46234/ccdcw2020.087>. 
CDC. Centers for Disease Control and Prevention. (2020)Use Personal Protective Equipment (PPE) When Caring for Patients with Confirmed or Suspected COVID-19. EUA. https://www.cdc.gov/coronavirus/2019-ncov/index.html?CDC_AA_refVal=https\%3A\%2F\%2Fwww.cdc.gov\%2Fcoronavir us\%2Findex.html

Chan, J. F., Kok, K. H., Zhu, Z., Chu, H., To, K. K., Yuan, S., \& Yuen, K. Y. (2020). Genomic characterization of the 2019 novel human-pathogenic coronavirus isolated from a patient with atypical pneumonia after visiting Wuhan. Emerging microbes \& infections, 9(1), 221-236.

ECDC. European Center for Disease Prevention and Control. (2020). ECDC Technical Report. Infection prevention and control and preparedness for COVID19 in healthcare settings. <https://www.ecdc.europa.eu/en/publications-data/i nfection-prevention-and-control-and-preparedness-co vid-19-healthcaresettings>

Estrela, C. (2018). Metodologia Científica: Ciência, Ensino, Pesquisa. (3a. ed.), Artes Medicas, 725.

Gawande, A. (2020) Keeping the Coronavirus from Infecting Health-Care Workers. The New Yorker.

Holland, M., Zaloga, D. J., \& Friderici, C. S. (2020).COVID-19: Personal Protective Equipment (PPE) for the emergency physician. Vis J Emerg Med. 19:100740.

Honda, H. \& Kentaro, I. (2016) Personal Protective Equipment and Improving Compliance Among Healthcare Workers in High-Risk Settings. Curr Opin Infect Dis. 29(4):400-6.

Huang, C., Wang, Y., Li, X.,Ren, L., Zhao, J., Hu, Y., Zhang, L., Fan, G., Xu, J., Gu, X., Cheng, Z., Yu, T., Xia, J.,Wei, Y.,Wu, W., Xie, X.,Yin, W., Li, H., Liu, M. \& Cao, Bin. (2020). Clinical features of patients infected with 2019 novel coronavirus in Wuhan, China. The Lancet. 395. 10.1016/S01406736(20)30183-5.

INAIL. Istituto Nazionale Per L'assicurazione Contro Gli Infortuni Sul Lavoro. (2020). Ministero della Salute. Direzione Generale Della Prevenzione Sanitaria Ufficio 5 Prevenzione Delle Malattie Trasmissibili E Profilassi Internazionale. Interim indications for rational use of protections for infection with SARS-CoV-2 in health and socio-health activities (assistance to subjects affected by COVID-19) in the current SARS-COV-2 emergency scenario - updated to <http://www.trovanorme.salute.gov.it /norme/renderNormsanPdf?anno=2020\&codLeg=73770\&parte=1\%20\&serie=null>.

Koh, D. (2020).Occupational risks for COVID-19 infection. Occup Med (Lond); 70(1):3-5.

Lipsitch, M., Swerdlow, D. L., \& Finelli, L. (2020). Defining the Epidemiology of Covid-19 - Studies Needed. The New England journal of medicine, 382(13), 1194-1196.

Ministério da Saúde -MS. (2020) Recomendações de proteção aos trabalhadores dos serviços de saúde no atendimento de COVID-19 e outras síndromes gripais. Brasília 2020.

Ministério do Trabalho-MTB. NR 6 Equipamentos de proteção individual [Internet]. Brasilia: MTB; 2020. < http://trabalho.gov.br/seguranca-e-saude-notrabalho/normatizacao/normas-regulamentadoras.>

Organização Internacional do Trabalho - OIT. Convenção 155. (1981). 〈http://www.conjur.com.br/dl/convencao- oit-155.pdf>. <http://www.conjur.com.br/dl/convencao- oit-155.pdf>.

Organização Pan-Americana de Saúde - OPAS- Brasil. OMS afirma que COVID-19 é agora caracterizada como pandemia. <https://www.paho.org/bra/index.php?option=com_content\&view=article\&id=6120:oms-afirma-que-covid-19-e-agora-caracterizada-comopandemia\&Itemid $=812>$.

PanAmerican Health Organization (PAHO)/World Health Organization (WHO), (2020).COVID-19 has infected some 570.000 health workers and killed 2500 in the Americas.2020.

Ran, L., Chen, X., Wang, Y., Wu, W., Zhang, L. \& Tan, X. (2020). Risk Factors of Healthcare Workers With Coronavirus Disease 2019: A Retrospective Cohort Study in a Designated Hospital of Wuhan in China. Clinical infectious diseases: an official publication of the Infectious Diseases Society of America, 71(16), 2218-2221.

Teixeira, C. F. S., Soares, C. M, Souza, E. A, Lisboa, E. S, Pinto, I. C. M, Andrade, L. R. \& Espiridião, M. A (2020). A saúde dos profissionais de saúde no enfrentamento da pandemia de Covid-19. Ciência \& Saúde Coletiva [online]. 25(9): 3465-3474.

Van Doremalen, N., Bushmaker, T., Morris, D. H., Holbrook, M. G., Gamble, A., Williamson, B. N., Tamin, A., Harcourt, J. L., Thornburg, N. J., Gerber, S. I., Lloyd-Smith, J. O., de Wit, E. \& Munster, V. J. (2020). Aerosol and Surface Stability of SARS-CoV-2 as Compared with SARS-CoV-1. The New England journal of medicine, 382(16), 1564-1567.

WHO. World Health Organization. (2020). IHR procedures concerning public health emergencies of international concern (PHEIC). <http://www.who.int /ihr/procedures/pheic/en/>.

WHOa. (2020). World Health Organization Keep health workers safe to keep patients safe: WHO-Geneva.

WHOb. (2020).World Health Organization. Rational use of personal protective equipment for coronavirus disease (COVID-19): Interim guidance, World Health Organization; 2020. https:// apps.who.int/iris/bitstream/handle/10665/331215/WHO-2019-nCov-IPCPPE_use-2020.1-eng. pdf?sequence=1\&isAllowed $=\mathrm{y}$

World O’ Meters - Brasil. 2020. <https://www.worldometers.info/coronavirus/country/brazil/>.

Xiang, Y. T., Yang, Y., Li, W., Zhang, L., Zhang, Q., Cheung, T. \& Ng, C. H. (2020). Timely mental health care for the 2019 novel coronavirus outbreak is urgently needed. The lancet. Psychiatry, 7(3), 228-229. 
Research, Society and Development, v. 10, n. 7, e55010716809, 2021

(CC BY 4.0) | ISSN 2525-3409 | DOI: http://dx.doi.org/10.33448/rsd-v10i7.16809

Yan, Y., Chen, H., Chen, L., Cheng, B., Diao, P., Dong, L., Gao, X., Gu, H., He, L., Ji, C., Jin, H., Lai, W., Lei, T., Li, L., Li, L., Li, R., Liu, D., Liu, W., Lu, Q., Shi, Y., \& Li, H. (2020). Consensus of Chinese experts on protection of skin and mucous membrane barrier for health-care workers fighting against coronavirus disease 2019. Dermatologic therapy, 33(4), e13310.

Zhang, Z., Yao, W., Wang, Y., Long, C. \& Fu, X. (2020). Wuhan and Hubei COVID-19 mortality analysis reveals the critical role of timely supply of medical resources. The Journal of infection, 81(1), 147-178. 